\title{
PLATAFORMA SIVEA - UNISON, COMO HERRAMIENTA TIC COADYUVANTE EN HACER EFECTIVO EL DERECHO HUMANO A LA EDUCACIÓN INCLUSIVA Y EQUITATIVA DE CALIDAD PARA TODOS ${ }^{1}$. \\ Adria Velia González Beltrones, Rafael Ramírez Villaescusa, ${ }^{2}$ María del Carmen Morales Tostado ${ }^{3}$, Hermes Ruiz Paredes. ${ }^{4}$
}

Sumario:I.Introducción.II.Antecedentes del derecho humano a la educación de calidad DHEC.III. Compromisos de México al 2030.IV. Oportunidad de accionar de las Instituciones de Educación IE V. Conclusiones

\section{Resumen}

En este documento se propone que Mediante un Plan Estratégico la Universidad de Sonora UNISON conjunte su Capital Humano y sus Recursos Materiales, especificamente, la Herramienta TIC (plataforma sivea) conforme al marco ampliado de la Agenda Mundial Educación 2030, para contribuir de manera permanente, con el Estado Mexicano, al objetivo de concretar --- si se repica la experiencia en las restantes 31 entidades federativas--- el derecho humano a la educación inclusiva y equitativa de calidad para todos y el compromiso de México con la UNESCO en tiempo y forma.

Palabras clave: educación de calidad inclusiva y equitativa, alianzas universidades, gobierno y sociedad

\footnotetext{
${ }^{1}$ La versión sintética de este trabajo, aceptado el primero de abril de 2019, se presentó en el XXIV Congreso Internacional de Tecnologías para la Educación y el Conocimiento (InterEstraTIC 2019)UNED, Facultad de Educación, Madrid, España,27 de junio de 2019

2 Docentes investigadores del Departamento de Derecho, DCS, URC, Universidad de Sonora, correos electrónicos: adria.gonzalez@unison.mx; rafael.ramirez@unison.mx

${ }^{3}$ Docente investigadora del Departamento de Contabilidad, DCEA,URC,Universidad de Sonora, correo electrónico: mcmorales@pitic.uson.mx

${ }^{4}$ Estudiante de Doctorado en Administración Pública Instituto Sonorense de Administración Pública ISAPAC
} 
Plataforma SIVEA-Unisón, como herramienta TIC coadyuvante en hacer efectivo el derecho humano a la educación inclusiva y equitativa de calidad para todos.

Año 11, Número 20 enero-junio 2019

Adria Velia González Beltrones, Rafael Ramírez Villaescusa, María del Carmen Morales Tostado. Hermes Ruiz Paredes.

\section{Abstract}

In this document It is proposed that through a Strategic Plan the University of Sonora UNISON combine its Human Capital and its Material Resources specifically the ICT Tool (sivea platform) in accordance with the expanded framework of the World Education Agenda 2030, to contribute permanently with the Mexican State to the objective of specifying --- if the experience in the remaining 31 states is repeated --- the human right to quality inclusive and equitable education for all and Mexico's UNESCO commitment in a timely manner.

Keywords: inclusive and equitable quality education, university, government and society alliances

\section{INTRODUCCIÓN}

En la Declaración de Incheon aprobada en el Foro Mundial sobre la Educación en mayo de 2015 se reconoció que la educación es fundamental para el éxito de los 17 objetivos aprobados y encomendó, a la UNESCO, como organismo de las Naciones Unidas especializado en educación, que dirigiera y coordinara la agenda Educación 2030 con sus asociados. Se estableció además, una hoja de ruta para conseguir las diez metas del objetivo de la educación que es el Marco de Acción Educación 2030, aprobado en noviembre de 2015, el cual ofrece orientación a los gobiernos y a sus socios para convertir los compromisos en acción.

Objetivo

En el presente documento se analiza la viabilidad de conjuntar, mediante un Plan Estratégico acorde a la Agenda Mundial Educación 2030 AME 2030, el Capital Humano y Recursos Materiales,en específico, la Herramienta TIC (plataforma sivea)de la Universidad de Sonora para coadyuvar de manera permanente con el Estado Mexicano 5 a cumplir su compromiso con la UNESCO al 2030 de los Objetivos de Desarrollo Sostenible ODS 4 y 5 en la implementación de la educación inclusiva y equitativa de calidad para todos".

\footnotetext{
${ }^{5}$ En lo concerniente a la educación en todos los niveles dentro del Estado de Sonora
} 


\section{Contexto}

Por una parte,en nuestra Universidad de Sonora UNISON se cuenta con una heramienta TIC (plataforma sivea ${ }^{6}$ ) si bien perfectible, a la cual tenemos acceso los actores de la institución, por otra parte,en época de austeridad económica y recortes presupuestales, es propicio recordar la conveniencia de revalorar, además de los recursos materiales, el capital humano ${ }^{7}$ de que dispone la institución para sumarse--- conjuntamente con sus homólogas de las restantes entidades federativas EF---mediante un Plan Estratégico, a la tarea titánica ---del Estado Mexicano através de los ordenes de gobierno federal,locales y municipales-- que significa, alcanzar, en diez años más (2030) cumplir, con hacer efectivo el derecho humano a la educación de calidad para todos, en todos los niveles (ODS 4 y 5).

\section{Hipótesis}

Conjuntando ambos recursos [CH+RM(HTIC)] PE AME2030, mediante un Plan Estratégico, teniendo como marco el nuevo ámbito ampliado de la Agenda Mundial Educación $2030^{\circ}$, es factible, que la UNISON contribuya de manera

${ }^{6}$ En la Universidad de Sonora contamos a partir del 2008 con la plataforma digital Sistema Virtual de Enseñanza Aprendizaje SIVEA que, en una primera fase, ha sido y es utilizada por la Universidad de Sonora (UNISON) entre otros propósitos, tanto como apoyo didáctico en un modelo educativo presencial, tanto cuanto, herramienta digital de apoyo académico-administrativo entre las autoridades universitarias, sus estudiantes y su personal académico y administrativo. A la fecha no se ha transitado a la modalidad de educación a distancia o por lo menos semipresencial(blended).

${ }^{7}$ La Universidad de Sonora cuenta actualmente con 2558 Académicos de los cuales 1275 son de asignatura y 1283 de carrera ; cuenta con 30,000 Alumnos : 28,702 de Licenciatura y 1219 de Posgrado distribuidos en 3 Unidades Regionales Académicas, 6 Campus, 11 Divisiones Académicas, su oferta Académica son 50 licenciaturas y 50 Posgrados 4 Academias de Arte y 10 Cursos de Idiomas Vide: Universidad de Sonora UNISON,Plan de Desarrollo Institucional 2017-2021, Numeralia 2019[en línea]Disponible en : https://www.unison.mx/numeralia/; consultada mayo 2019.

${ }^{8}$ que se extiende desde el aprendizaje en la primera infancia hasta la educación y la formación de jóvenes y adultos; que prima la adquisición de habilidades para trabajar y que entre otros, subraya la importancia de la educación de la ciudadanía en un mundo plural e interdependiente;centrado en la inclusión, la equidad y la igualdad entre ambos sexos y con la valerosa pretensión de garantizar resultados de calidad en el aprendizaje para todos, a lo largo de toda la vida. 
Plataforma SIVEA-Unisón, como herramienta TIC coadyuvante en hacer efectivo el derecho humano a la educación inclusiva y equitativa de calidad para todos.

Año 11, Número 20 enero-junio 2019

Adria Velia González Beltrones, Rafael Ramírez Villaescusa, María del Carmen Morales Tostado. Hermes Ruiz Paredes.

permanente con la Secretaría de Educación y Cultura SECSON del gobierno del estado de Sonora,y demas instancias gubernamentales federal y/o locales competentes, al objetivo de lograr que todos y cada uno de ios centros de educación de todos los niveles cuenten con: Infraestructura escolar, materiales y métodos educativos,personal docente y directivo y organización escolar eficaces, para resolver la

\section{Problemática}

De hacer efectivo el derecho humano a la educación de calidad para todos en todos los niveles (ODS 4 y 5). Para ello la

Discusión se centra, en términos de la necesidad de adopción, por parte de la UNESCO, de medidas de solución realistas y efectivas- a la problemática que en este trabajo nos ocupa--acorde a la realidad individual de cada país aunado al esfuerzo colectivo al efecto.

\section{ANTECEDENTES}

En México,los derechos humanos como "conjunto de prerrogativas inherentes de que goza toda persona," se encuentran reconocidos en su Constitución Política y en los Tratados Internacionales en los que el Estado Mexicano es parte. Ciertamente las tres generaciones históricas ${ }^{9}$ de derechos humanos, se han incorporado paulatinamnete en la Constitución Política de los Estados Unidos Mexicanos CPEUM y a partir de 2011 forman parte del bloque constitucional los Tratados Internacionales de Derechos Humanos reconocidos por

\footnotetext{
${ }^{9}$ Por su evolución, pueden distinguirse tres generaciones de derechos humanos presentes en la historia de la humanidad.Los de $1^{\text {a }}$ generación:Civiles y politicos(derechos a la vida, al nombre, a la nacionalidad; a la igualdad, a la libertad, a la seguridad; a ser oídas en juicio por un tribunal independiente e imparcial, a que se presuma su inocencia mientras no se pruebe su culpabilidad; las libertades de tránsito, de religión, de pensamiento, de expresión, de reunión y de asociación; a participar en el gobierno de su país, a casarse y fundar una familia; derechos de la infancia; y se prohíben la discriminación, la esclavitud, la tortura o los tratos crueles, inhumanos o degradantes.Los de 2a. generación:Económicos, sociales y culturales. Los de 3a. Generación: De los pueblos o de solidaridad y ambientales (conservación en el equilibrio para la protección y explotación naturales; derecho al trabajo, a la seguridad social, a la educación, a la salud, a la cultura y a un ambiente sano.
} 
México. En este punto cabe mencionar, que si bien en la mayoría de los paises, el derecho humano a la educación, ha estado presente, desde la 1a. generación, también es dable recordar que ha evolucionado su forma de observancia por lo gobiernos de los países miembros de la UNESCO. Como se menciona líneas arriba,en México, el derecho a la educación se ha reconocido en la Constitución Política de los Estados Unidos Mexicanos desde 1917 en el artículo $3^{\circ}$ (1a. y 2a. Generación)y sus reformas subsecuentes, entre otras, las de incluir a partir de $1948^{10}$ los derechos humanos de índole económica social y cultural ${ }^{11}$ (ver líneas debajo Tabla 1).

10 La Declaración Universal de Derechos Humanos, aprobada por la Asamblea General de la Organización de Naciones Unidas en 1948, en sus 30 artículos consagró la protección de derechos civiles y políticos, así como de derechos económicos, sociales y culturales. Estos derechos fueron a su vez protegidos en dos tratados internacionales, el Pacto Internacional de Derechos Civiles y Políticos y el Pacto Internacional de Derechos Económicos, Sociales y Culturales, ambos aprobados en 1966 y obligatorios para México desde 1981. Desde el proceso de elaboración de los dos Pactos Internacionales, la Asamblea General de Naciones Unidas, en 1950, en su Resoluciónn 421 E (V), señaló que el goce de derechos civiles y políticos y el de los derechos económicos, sociales y culturales "están vinculados entre sí y se condicionan mutuamente.Los dos Pactos Internacionales, que se procuró contemplaran disposiciones comunes, cada uno en su artículo 2 señala el compromiso "a garantizar el ejercicio de los derechos que en él se enuncian, sin discriminación alguna por motivos de raza, color, sexo, idioma, religión, opinión política o de otra índole, origen nacional o social, posición económica, nacimiento o cualquier otra condiciónn social'. Asimismo, en el artículo 3 estableció el compromiso a asegurar a los hombres y a las mujeres igual título a gozar de todos los derechos civiles y políticos y económicos, sociales y culturales enunciados en cada Pacto. De ello se desprende que todas las personas son titulares de todos los derechos humanos, que se conoce como el principio de universalidad.

${ }^{11}$ reconocidos actualmente de manera explícita los siguientes: "a la protección de las manifestaciones de los pueblos indígenas (artículo 2o., apartado A); acceso a los beneficios del progreso científico (artículo 3o., fracción III); participación en la vida creativa y libertad para la actividad creativa (artículo 4o., duodécimo párrafo); al igual que la protección a los intereses morales y materiales correspondientes a las producciones científicas, literarias o artísticas (artículo 28, décimo párrafo). A lo expuesto, cabe añadir que el duodécimo párrafo del artículo 40 Constitucional establece una cláusula general de respeto y protección de todos los derechos 
Plataforma SIVEA-Unisón, como herramienta TIC coadyuvante en hacer efectivo el derecho humano a la educación inclusiva y equitativa de calidad para todos.

Año 11, Número 20 enero-junio 2019

Adria Velia González Beltrones, Rafael

Ramírez Villaescusa, María del Carmen Morales Tostado. Hermes Ruiz Paredes.

Tabla 1 Evolución en México del DH a la Educación

\begin{tabular}{|c|c|c|c|c|}
\hline Documento & AÑO & $1^{\mathrm{a}} \mathrm{G}$ & $2^{a} G$ & $3^{a} \mathrm{G}$ \\
\hline CPEUM & 1917 & Art.3음 & & \\
\hline CPEUM & 1948 & & 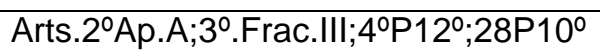 & \\
\hline CPEUM/TIDH & 2011 & & & $\begin{array}{l}\text { Arts. } 2^{\circ} A p . A ; 3^{\circ} ; \text { Frac.III; } \\
4^{\circ}=2^{\circ} ; 28 \mathrm{P} 10^{\circ}\end{array}$ \\
\hline
\end{tabular}

En cuanto al derecho a la educación de 3a. Generación, a partir de la Reforma Constitucional de $2013^{12}$ se introdujo el reconocimiento de la educación de calidad como un derecho humano que, como tal, debe cumplir con los cuatro principios de los derechos humanos: universalidad, indivisibilidad, interdependencia y progresividad ${ }^{13}$

\section{Marco Teórico}

culturales, de lo que se permite concluir que existe un sistema jurídico consistente de protección a tales derechos en nuestro orden nacional.

${ }^{12}$ En el 2012 se declaró obligatoria la educación media superior en el país.

13 Vide Comisión Nacional de Derechos Humanos CNDH,Los principios de universalidad, interdependencia, indivisibilidad y progresividad de los derechos humanos, Ed.,CNDH México, 2018, pp.5 "Por su parte, el Pacto Internacional de Derechos Económicos, Sociales y Culturales, en su artículo 2 señala el compromiso "a adoptar medidas, tanto por separado como mediante la asistencia y la cooperación internacionales, especialmente económicas y técnicas, hasta el máximo de los recursos de que disponga, para lograr progresivamente, por todos los medios apropiados, inclusive en particular la adopción de medidas legislativas, la plena efectividad de los derechos aquí reconocidos". El Comité de Derechos Económicos, Sociales y Culturales, en su Observación General número 3, de 1990, señaló en su párrafo 9 que la "progresiva efectividad" implica un reconocimiento de que la plena efectividad de todos los derechos económicos, sociales y culturales podrá lograrse en un periodo de tiempo, pero impone la obligación de proceder lo más expedita y eficazmente posible para lograr ese objetivo. Desde 1977, la Asamblea General de las Naciones Unidas, en su resolución 32/130, reafirmó que "todos los derechos humanos y libertades fundamentales están interrelacionados y son indivisibles", que se han caracterizado como los principios de interdependencia e indivisibilidad. De esta forma, en 1993, en la Convención Mundial de Derechos Humanos se aprobó la Declaración y Programa de Viena, que en su numeral 5 precisó "Todos los derechos humanos son universales, indivisibles e interdependientes y están relacionados entre sí". 
De todos es sabido que el uso de las Tecnologías de la Información y la Comunicación TIC en educación, ha marcado una pauta en la enseñanza y en la formación de docentes y discentes. Desde fines del pasado Siglo XX y lo que va del presente, acorde a De Pablos, J. y Jiménez se ha pasado del objetivo de aprender las TIC a utilizarlas como herramientas de aprendizaje ${ }^{14}$. Asimismo, es de reconocerse en definitiva, que el uso de las TIC amplía las posibilidades de llevar a cabo el proceso enseñanza-aprendizaje tradicional presencial, o bien, pueden mejorar los métodos ya empleados, como es el caso de la modalidad educativa a distancia. ${ }^{15}$

Para efectos del tema que nos ocupa resulta conveniente clarificar la importancia teórica,del concepto de educación a distancia, en el que los medios son las TIC. En palabras de Clark ${ }^{16}$ la educación a distancia enfatiza la separación del profesor - alumno, durante el proceso instruccional, sin embargo, se emplean los medios (TIC) como unión entre el profesor, el estudiante y los contenidos del curso.

Por su parte, Garrison, ${ }^{17}$ refiere que la educación a distancia implica que la mayor parte de la comunicación entre el profesor y el estudiante ocurre de forma no

\footnotetext{
${ }^{14}$ De Pablos Pons, J. y Jiménez Cortés, R. (2007). Buenas prácticas con TIC apoyadas en las Políticas Educativas: claves conceptuales y derivaciones para la formación en competencias ECTS, Revista Latinoamericana de Tecnología Educativa, 6(2), 15-28 [en línea] No Disponible actualmente vide en: http://campusvirtual.unex.es/cala/editio/index.php?journal=relatec\&page=article\&o p=viewFile\&path[] $=345 \&$ path[] $=293$

${ }^{15} \mathrm{La}$ que anteriormente se realizaba mediante correspondencia o en la modalidad del sistema de enseñanza abierto SEA en el que por medio de manuales por asignatura se guiaba el autoaprendizaje interactuando docente-estudiante mediante tutorías presencialmente una vez al mes para realizar exámenes o aclarar dudas. UNED pionera en Ibero América cumplirá el próximo mes de agosto sus primeros 47 años.

16 Clark R. "Reconsidering Research on Learning from Media," Review of Educational Research, 1983, 53, (4). pp. 445-459.

17 Vide:Garrison, D. R. Understanding Distance Education: A Framework for the Future. London: Routledge,1989, in Mclsaac, M.S. \& Gunawardena, C.N. Distance Education. In D.H. Jonassen, ed. Handbook of research for educational communications and technology: a project of the Association for Educational
} 
Plataforma SIVEA-Unisón, como herramienta TIC coadyuvante en hacer efectivo el derecho humano a la educación inclusiva y equitativa de calidad para todos.

Año 11, Número 20 enero-junio 2019

Adria Velia González Beltrones, Rafael Ramírez Villaescusa, María del Carmen Morales Tostado. Hermes Ruiz Paredes.

adyacente. El mismo autor hace alusión a que este tipo de educación, debe considerar dos vías de comunicación entre el profesor y el estudiante, con el fin de sobrellevar el proceso educativo, mediante el uso de las TIC. En este tenor, la definición de educación a distancia que se adecua mas al desarrollo de nuestro tema, es la descrita por García18 la que se concibe como "el proceso educativo realizado por una organización en forma masiva, por medio de un sistema tecnológico de información y comunicación multidireccional, apoyado en la acción sistemática y conjunta de recursos didácticos y de tutoría, que separados físicamente de las y los estudiantes, favorece el auto-aprendizaje de forma asincrónica y/o sincrónica ${ }^{19}$ ".

Communications and Technology. 1996, pp.403-437. New York: Simon \& Schuster Macmillan

18 Vide: García, L., La educación a distancia: de la teoría a la práctica. Barcelona, España, Editoral Ariel,2001, en la que concibe a la educación a distancia como "un sistema tecnológico de comunicación multidireccional, que puede ser masivo, basado en la acción sistemática y conjunta de recursos didácticos y el apoyo de una organización y tutoría, que separados físicamente de los estudiantes,propician un aprendizaje independiente"; Vide: versión modificada por González B.A., Avendaño E.A., Olavarrieta C. M., Maya R.J. (autores) de: Plataformas virtuales de aprendizaje y gestión académica: SIBAL y SIVEA, Herramientas de sustentabilidad en su utilización presencial y a distancia Estudio de Caso: Programa de Preparatoria COBACH - Programa de Licenciatura en Derecho UNISON,en Memorias del XVIII Congreso Internacional de Tecnologías para la Educación y el Conocimiento y $\mathrm{V}$ de Pizarra Digital: Interculturalidad, Estrategias y Tecnologías, Facultad de Educación de la UNED,Madrid, España, 26, 27, 28 y 29 de Junio de 2013,pp.2

${ }^{19}$ Se considera que las herramientas de comunicación sincrónica son aquéllas que, en cualquier curso de formación en línea-online-, permiten el proceso de comunicación en tiempo real entre alumnos y tutores; es decir, que ambos deben estar conectados e interactuar al mismo tiempo. Ejemplos de estas herramientas de comunicación sincrónica son: los chats, los foros, las videoconferencias, las webconference y la pizarra digital, entre otros. En cuanto a las herramientas de comunicación asincrónica son aquéllas que, en cualquier curso de formación en línea- online-, el proceso de comunicación entre alumnos y tutores no se realiza en tiempo real; es decir, que ambos no están conectados ni interactúan al mismo tiempo. Ejemplos: mensajería por correo electrónico, tareas en plataforma y los foros asincrónicos, entre otros. 
En esta misma línea, Gámez, ${ }^{20}$ enfatiza que la educación a distancia permite acoplar el aprendizaje continuo a la particular circunstancia de vida de cada persona, una vez que ésta explore todas sus opciones podrá decidir si tal modalidad es la mejor opción. La educación a distancia se presenta como una forma flexible de aprendizaje, que ofrece opciones de acuerdo con las condiciones del estudiante, con base en esto, se pueden desarrollar aprendizajes de manera individual o en grupo, reuniéndose en un aula, una casa o comunicándose con otros alumnos e instructores a través de un espacio virtual.

\section{METODOLOGÍA}

Hoy en día, las Tecnologías de Información y Comunicación son recursos utilizados cotidianamente por una porción significativa de la población mundial, de manera que inciden en diversos campos de la actividad individual y colectiva del hombre moderno,a ello ha contribuido la adquisición masiva de teléfonos celulares inteligentes al alalcance de prácticamente todo bolsillo y la expansión del acceso a la internet. Desde esta perspectiva, en relación a los compromisos adquiridos por el Estado Mexicano, de hacer efectivo, el derecho humano a la educación de calidad para todos al 2030, y de nuestra propuesta, de coadyuvancia, en alcanzar tal objetivo,de las Instituciones de Educación IE(de niveles medio básico IEMB,medio superior IEMS o superior IES, de nuestra entidad Sonora y las de sus homólogas de las restantes EF) se desprende que, la educación es un campo, en el cual, la incorporación de las TIC obliga no solo a repensar la práctica docente y las metodologías didácticas en el sistema tradicional presencial21, sino de manera

\footnotetext{
${ }^{20}$ Gámez, J. Evaluación del programa SIBAL como alternativa innovadora en la educación media superior. Tesis de Maestría no publicada. Universidad del Valle de México, Maestría en Educación,2010

${ }^{21}$ pues al introducir los recursos digitales y tecnológicos en las actividades escolares, se produce una alteración en la manera de realizar la práctica tradicional y formal de la educación, así como en los ejercicios y en la impartición de los contenidos de cada asignatura. Vide: González B.A. et al., Plataformas... op.cit., pp.3
} 
Plataforma SIVEA-Unisón, como herramienta TIC coadyuvante en hacer efectivo el derecho humano a la educación inclusiva y equitativa de calidad para todos.

Año 11, Número 20 enero-junio $2019 \quad$ Adria Velia González Beltrones, Rafael Ramírez Villaescusa, María del Carmen Morales Tostado Hermes Ruiz Paredes.

específica, en relación a la necesidad de nuestro país -México- de educar en forma masiva a ciertos sectores de la población, con una inversión menor en infraestructura material y en recursos humanos que posibiliten,democráticamente,el camino hacia el desarrollo económico, innovación educativa y cumplimiento de los compromisos de la Agenda Mundial de Educación 2030.

\section{COMPROMISOS DE MÉXICO AL 2030}

Corresponde en este punto, en primer término, listar de manera enunciativa mas no limitativa los compromisos adquiridos por México para asegurar el derecho humano a la educación acorde a la AME 2030, entre los que se encuentran los siguientes:

A. Cumbre Mundial de la Tierra realizada en Rió Agenda 21, capítulo 36 párrafo 3; Capítulo 3, párrafo 2 y capítulo 24, párrafo 3

"La Educación... deberá ser reconocida como un proceso por medio del cual los seres humanos y las sociedades pueden alcanzar su entero potencial. La educación...promueve el derecho sustentable y mejora la capacidad de las personas para manejar temas como medio ambiente y desarrollo Los gobiernos deberían tomar pasos activos para eliminar el analfabetismo.... y expandir la participación de la mujer en todos los ámbitos ...tanto en instituciones educacionales, como para promover la meta universal del acceso a una educación primaria y secundaria "

B. Cumbre Mundial para el Desarrollo Social de Copenhague Declaración de Copenhague, Compromiso 6

"Nos comprometemos a promover y a lograr los objetivos del acceso universal y equitativo a una educación de calidad... Formularemos y fortaleceremos estrategias nacionales con plazos precisos para erradicar el analfabetismo y universalizar la educación básica, que comprende la educación en la primera infancia, la educación primaria y la educación para analfabetos en todas las comunidades, ...intensificaremos $y$ coordinaremos el apoyo internacional a los programas de educación y salud basados en el respeto de la dignidad humana y centrados en la protección de todas las mujeres y niños....

C.Conferencia Mundial sobre la Mujer de Beijing Plataforma de Acción de Beijing, párrafos $69,80,81$, y 82

“La educación es un derecho humano y constituye un 


\section{BIOLEX}

instrumento indispensable para lograr los objetivos de la 
Plataforma SIVEA-Unisón, como herramienta TIC coadyuvante en hacer efectivo el derecho humano a la educación inclusiva y equitativa de calidad para todos.

Año 11, Número 20 enero-junio $2019 \quad$ Adria Velia González Beltrones, Rafael Ramírez Villaescusa, María del Carmen Morales Tostado, Hermes Ruiz Paredes.

igualdad, el desarrollo y la paz. La educación no discriminatoria beneficia tanto a las niñas como a los niños... Medidas que han de adoptar los gobiernos... Reducir la tasa de analfabetismo femenino por lo menos a la mitad de la tasa de 1990... Proporcionar acceso universal a la enseñanza primaria a las niñas, y procurar lograr la igualdad de género en la tasa de finalización de dicha enseñanza, para el año 2000...readiestramiento para las mujeres, en particular las jóvenes y las que retornen al mercado de trabajo...Establecer sistemas de educación y capacitación no discriminatorios..."

D. Conferencia Hábitat II de Estambul.Agenda de Hábitat Párr., 2.36 y 3. 43

"Nosotros. nos comprometemos a promover y a cumplir las metas planteadas para lograr un acceso igualitario universal y de la calidad en la educación... haciendo esfuerzos particulares para ratificar desigualdades relacionadas a las condiciones sociales $y$ económicas...sin distinción de ningún tipo por motivos de raza, origen, genero, edad, discapacidad, respetando y promoviendo nuestras propias culturas........

E. Síntesis de la Declaración mundial de educación para todos.Preámbulo y Art. 1 "...La educación es un derecho fundamental para todas las personas, mujeres, hombres, de todas las edades y en todo el mundo... Cada persona-niño, niña, joven y adulto debe beneficiarse de las oportunidades educacionales diseñadas para satisfacer sus necesidades básico...desarrollar sus plenas capacidades, vivir y trabajar en dignidad... mejorar la calidad de sus vidas, tomar decisiones. "

F. Afirmación de Amman 1996

"La educación da poder. Es la clave para el establecer y fortalecer la democracia y el desarrollo ...... derecho a la educación no es nada menos que el derecho a participar en el mundo moderno"

\section{OPORTUNIDAD DE ACCIONAR DE LAS IE}

En segundo término,corresponde plantear, la posible alianza de las IE como la UNISON y sus homólogas de las restantes Entidades Federativas EF, con las autoridades gubernamentales competentes, para que, mediante un Plan Estratégico las IE como la Universidad de Sonora UNISON conjunten su Capital Humano y respectivamente sus Herramientas TIC (plataforma sivea de la UNISON) 
[teniendo como marco el nuevo ámbito ampliado de la Agenda mundial Educación 203022] para contribuir de manera permanente con la Secretaría de Educación y Cultura del Gobierno del Estado de Sonora SECSON y demas instancias gubernamentales federal y/o locales competentes, al objetivo de lograr que todos y cada uno de los centros de educación de todos los niveles en Sonora cuenten con:Infraestructura escolar, materiales y métodos educativos, personal docente y directivo y organización escolar eficaces, para hacer realidad --- si se repica la experiencia en las 31 entidades federativas--- el derecho humano a la educación de calidad para todos y con ello Mexico cumpla en tiempo y forma su compromiso $[\mathrm{IE}>(\mathrm{CH}+\mathrm{RM}(\mathrm{HTIC}) \mathrm{PE} / \mathrm{AME2030}=\mathrm{DHEC} \vee 2030]$.

En tercer término cabe mencionar como antecedente de la viabilidad de la propuesta que hoy nos ocupa,la presentada hace 6 años ${ }^{23}$ del estudio de caso de la problemática de la enseñanza media superior y superior en México y concretamente en el estado de Sonora, en la que primeramente nos referimos a la herramienta del Sistema de Bachillerato en Línea SIBAL-que ofrece educación a distancia media superior- a través de los recursos de las TIC. Posteriormente, se abordó la del Sistema Virtual de Enseñanza Aprendizaje SIVEA - que en una primera fase ofrece apoyo didáctico a la educación superior presencial en la Universidad de Sonora UNISON-y al contrastar sus posibilidades, se llegó a la parte final del trabajo de investigación, en la cual se desarrollaron propuestas para fortalecer las áreas débiles de ambos sistemas con base en literatura revisada,

${ }^{22}$ que se extiende desde el aprendizaje en la primera infancia hasta la educación y la formación de jóvenes y adultos; que prima la adquisición de habilidades para trabajar y que entre otros, subraya la importancia de la educación de la ciudadanía en un mundo plural e interdependiente;centrado en la inclusión, la equidad y la igualdad entre ambos sexos y con la valerosa pretensión de garantizar resultados de calidad en el aprendizaje para todos, a lo largo de toda la vida.

${ }^{23}$ en la edición XVIII del Congreso Internacional de Tecnologías para la Educación y el Conocimiento y V de Pizarra Digital: Interculturalidad, Estrategias y Tecnologías de la UNED, 
Plataforma SIVEA-Unisón, como herramienta TIC coadyuvante en hacer efectivo el derecho humano a la educación inclusiva y equitativa de calidad para todos.

Año 11, Número 20 enero-junio 2019

Adria Velia González Beltrones, Rafael Ramírez Villaescusa, María del Carmen Morales Tostado. Hermes Ruiz Paredes.

dando sustento a las acciones proyectadas ${ }^{24}$. $\mathrm{Y}$ a partir de ello, se desglosaron propuestas de acciones de mejora, fundamento de la viabilidad de la alianza interinstitucional COBACH-UNISON, con el doble propósito de enriquecer su desarrollo impulsando su continuidad e impacto en la educación a distancia y con ello abatir el rezago educativo en educación media superior y superior en el Estado de Sonora.

Ahora bien,en cuarto lugar, en este Congreso consideramos propicio el plantear nuevamente, [en un contexto mas amplio, en cuanto a cobertura se refiere,la viabilidad de que las IE(Superior IES, Media Superior IEMS, y/o de Educación Media Básica IEMB) como la UNISON como se señalara lineas arriba, coadyuven con el Estado Mexicano, para hacer efectivo en México, el derecho humano a la educación de calidad para todos, en todos los niveles, en el marco de la Agenda Mundial de Educación 2030 y su Marco de Acción 2030 delineado por la UNESCO] .En este sentido enseguida, en quinto término cabe mencionar que el Instituto Nacional para la Evaluación de la Educación INEE ${ }^{25}$ desarrolló, basado en

\footnotetext{
24 Vide González Beltrones,A.V. et al, Plataformas virtuales de ...op.cit

${ }^{25}$ Hasta el 30 de mayo del presente año,con motivo de la entrada en vigor de la reforma constitucional en materia educativa publicada en el Diario Oficial de la Federación el 15 de mayo de 2019, que deroga diversas disposiciones de los artículos $3^{\circ}, 31$ y 73 de la Constitución Política de los Estados Unidos Mexicanos, finalmente se despejó la incognita en relación a si desaparecía o se transformaba la naturaleza jurídica del INEE, vide:1) nota relativa en :Político mx, de 18 de marzo de 2019, Nueva reforma educativa AMLO: adiós a INEE y a castigos para maestros 2019 [en línea] Disponible en : https://politico.mx/minuta-politica/minuta-politicacongreso/nueva-reforma-educativa-amlo-adiós-inee-y-castigos-para-maestros/ ; (consultado el 2 de abril de 2019; 2) Vide: Plataforma temporal que se conserva como un archivo histórico de las actividades del INEE[en línea]Disponible en: https://www.inee.edu.mx(consultado el 3 de junio de 2019).
}

3) Vide: Informe de la situación del INEE al 15 de mayo de 2019, Diario Oficial de la Federación DOF del 30 de mayo de 2019, Primera Sección,Coordinación de Administración del Organismo Público Descentralizado a que se refiere la fracción IX del Artículo 3o. de la Constitución Política de los Estados Unidos Mexicanos[en línea]Disponible en :

https://www.inee.edu.mx/wp-content/uploads/2019/05/Informe-INEE-DOF.pdf; (consultado el 3 de junio de 2019). En términos académicos, consideramos que tal medida es lamentablemente un desacierto ---de las autoridades temporales del 
las diversas evaluaciones efectuadas al Sistema Educativo, ${ }^{26}$ desde 2013 a 2018, la Agenda del Informe 2019, emitiendo,directrices, documentos ejecutivos de politica educativa recomendaciones para el sistema educativo, en los rubros de poblaciones vulnerables, recursos financieros, gestión escolar, infraestructura y equipamiento,currículo y materiales,docentes y directivos, relaciones intergubernamentales y evaluación del sistema educativo.

En este tenor, en sexto y séptimo lugar se considera que :Dicho Informe puede tomarse como punto de partida (radiografia por entidad federativa) para realizar, las Instituciones de Educación IE(Superior IES, Media Superior IEMS, y/o de Educación Media Básica IEMB) entre ellas la UNISON, conjuntamente con las autoridades gubernamentales competentes (Secretaría de Educación Pública SEP, Secretaría de Educación y Cultura del gobierno del estado de Sonora SECSON y/o sus homólogas de las restantes Entidades Federativas EF, Instituto Nacional para

poder político---el cambio de naturaleza jurídica del INEE, como Organo Autónomo de Carácter Nacional, con personalidad jurídica y patrimonio propio, con las atribuciones de evaluar la calidad, el desempeño y los resultados del Sistema Educativo Nacional en la educación preescolar, primaria, secundaria y media superior, a la de "un organismo público descentralizado no sectorizado (opdns) que coordinará el Sistema Nacional de Mejora Continua de la Educación"[es decir, un organismo público descentralizado, perteneciente al brazo paraestatal del ámbito de gobierno federal, estará realizando tácitamente funciones de Organo Autónomo Nacional, encubierto en un ropaje del brazo paraestatal del ámbito de gobierno federal,trastocando con ello la forma de estado adoptada por el Constituyente de 1917 (Art.40 CPEUM) como república representativa,democrática, laica, federal, en el que seguramente seguirá dictando las políticas y directrices en materia de educación en el ámbito nacional en detrimento de la autonomía y soberanía de las entidades federativas ya que tienen facultad concurrente con el ámbito de gobierno federal, en materia de educación preescolar, primaria, secundaria y media superior. 26 Informe anual se caracteriza por focalizar algún tema del sistema educativo. En el año 2006, el Consejo Técnico del INEE recomendó que los informes anuales se focalizaran en un tema particular. Así, en 2007 ... educación para poblaciones en contextos vulnerables y en 2008 ...un balance sobre avances en la calidad de la educación básica. Al decir del propio INEE en los últimos años, se ha centrado en la necesidad de definir referentes que permitan valorar los resultados de la medición de distintas dimensiones de la calidad de la educación... Ver modelo de evaluación de la calidad del Sistema Educativo Nacional en: INEE (2009). Panorama Educativo de México. 2008. México: INEE, pp. 25-31. 
Plataforma SIVEA-Unisón, como herramienta TIC coadyuvante en hacer efectivo el derecho humano a la educación inclusiva y equitativa de calidad para todos.

Año 11, Número 20 enero-junio 2019

Adria Velia González Beltrones, Rafael Ramírez Villaescusa, María del Carmen Morales Tostado. Hermes Ruiz Paredes.

la Evaluación de la Educación INEE ) el Plan Estratégico ${ }^{27}$ a seguir para hacer frente a los compromisos adquiridos por México en cuanto hacer realidad el derecho a la educación de calidad para todos, en todos los niveles, al 2030.

\section{CONCLUSIONES Y PROPUESTA}

Conforme a la Declaración de Incheon se reconoce que la educación es fundamental para el éxito de los 17 objetivos de desarrollo sostenible ODS aprobados en mayo de 2015 y se encomienda a la UNESCO, como organismo de las Naciones Unidas especializado en educación, dirigir y coordinar la Agenda Mundial de Educación 2030 con sus asociados.

El estado Mexicano adquirió la obligación de cumplir con varios indicadores para asegurar el el derecho humano a la educación de calidad para todos (ODS 4 y 5) en diez años más, 2030, acorde a la AME 2030.

Dada la importancia teórica,del concepto de educación a distancia, en el que los medios son las TIC, la definición de educación a distancia que se adecua mas al tema que nos ocupa, es la descrita por García ${ }^{28}$ la que se concibe como "el proceso educativo realizado por una organización en forma masiva, por medio de un sistema tecnológico de información y comunicación multidireccional, apoyado en la acción sistemática y conjunta de recursos didácticos y de tutoría, que separados físicamente de las y los estudiantes, favorece el auto-aprendizaje de forma asincrónica y/o sincrónica.

${ }^{27}$ Cabe añadir ---como séptimo término--- que de los puntos claves que, según los expertos, todo Plan Estratégico debe contener, a partir del diagnóstico previo realizado por el INEE, acorde a su Informe 2019, solo tendría que trabajarse en los ejes estratégicos de interacción de las IE y autoridades intergubernamentales con sus recursos y herramientas materiales disponibles, específicamente las TIC, adicionados con sus respectivos capitales humanos para operar el PE y la ruta crítica a seguir por año calendario, acorde a la definición previa de objetivos y acciones a realizar por las IE para cumplir con los indicadores de evaluación identificados previamente por la (AME y Marco de Acción Educación)2030.

Vide García ....op.cit 
En este tenor, dado que el Instituto Nacional para la Evaluación de la Educación INEE desarrolló, basado en las diversas evaluaciones efectuadas al Sistema Educativo, desde 2013 a 2018,la Agenda del Informe 2019, dicho Informe puede tomarse como punto de partida (radiografia por entidad federativa) para realizar [la UNISON en alianza con las Instituciones de Educación IE(Superior IES, Media Superior IEMS, y/o de Educación Media Básica IEMB de todo el pais ), con las autoridades gubernamentales competentes de orden federal,local y municipal] el Plan Estratégico ${ }^{29}$ a seguir para hacer frente a los compromisos adquiridos por México en cuanto hacer realidad el derecho a la educación de calidad para todos, en todos los niveles, al 2030.

${ }^{29}$ de los puntos claves que, según los expertos, todo Plan Estratégico debe contener, a partir del diagnóstico previo realizado por el INEE, acorde a su Informe 2019, solo tendría que trabajarse en los ejes estratégicos de interacción de las IE y autoridades intergubernamentales con sus recursos y herramientas materiales disponibles, específicamente las TIC, adicionados con sus respectivos capitales humanos para operar el PE y la ruta crítica a seguir por año calendario, acorde a la definición previa de objetivos y acciones a realizar por las IE para cumplir con los indicadores de evaluación identificados previamente por la (AME y Marco de Acción Educación)2030. 
Plataforma SIVEA-Unisón, como herramienta TIC coadyuvante en hacer efectivo el derecho humano a la educación inclusiva y equitativa de calidad para todos.

Año 11, Número 20 enero-junio 2019

Adria Velia González Beltrones, Rafael Ramírez Villaescusa, María del Carmen Morales Tostado. Hermes Ruiz Paredes.

\section{Bibliografia y Webgrafia}

Agenda Mundial Educación 2030[en línea]Disponible en:

https://es.unesco.org/themes/liderar-agenda-mundial-educacion-2030

Clark.,R., "Reconsidering Research on Learning from Media". Review of Educational Research, 53, (4). 1983

Comisión Nacional de Derechos Humanos, Los principios de universalidad, interdependencia, indivisibilidad y progresividad de los derechos humanos, Ed., CNDH México, 2018

Diario Oficial de la Federación DOF, Primera Sección,Jueves 30 de mayo de 2019, Coordinación de Administración del Organismo Público Descentralizado a que se refiere la fracción IX del Artículo 3o. de la CPEUM

Gámez, J., Evaluación del programa SIBAL como alternativa innovadora en la educación media superior. Tesis de Maestría no publicada. Universidad del Valle de México Maestro en Educación,2010

García, L., La educación a distancia: de la teoría a la práctica. Barcelona, España, Editoral Ariel,2001.

Garrison, D. R., Understanding Distance Education: A Framework for the Future. London: Routledge,1989

Instituto Nacional para la Evaluación de la Educación INEE, Derecho a la Educación en México. Informe 2009, Ed.INEE,México 2010

Derecho a la Educación en México. Informe 2019, Ed.INEE,México 2019

Plataforma temporal que se conserva como un archivo histórico de las actividades del INEE [en línea]Disponible en: https://www.inee.edu.mx

Instituto Nacional de Estadística y Geografía INEGI, Perfil Sociodemográfico. II Conteo de Población y Vivienda México2005.,

González Beltrones,A.V. et al, Plataformas virtuales de aprendizaje y gestión académica: SIBAL, y SIVEA, Herramientas de sustentabilidad en su utilización presencial y a distancia Estudio de Caso: Programa de Preparatoria COBACH - Programa de Licenciatura en Derecho UNISON, en 
Memorias delXVIII Congreso Internacional de Tecnologías para la Educación y el Conocimiento y V de Pizarra Digital: Interculturalidad, Estrategias y Tecnologías, Madrid, España, 26, 27, 28 y 29 de Junio de 2013.

Político mx, de 18 de marzo de 2019, Nueva reforma educativa AMLO: adiós a INEE y a castigos para maestros 2019 [en línea] Disponible en : https://politico.mx/minuta-politica/minuta-politica-congreso/nueva-reformaeducativa-amlo-adiós-inee-y-castigos-para-maestros/

Recibido 28 de febrero de 2019 Aceptado $1^{\circ}$ de abril de 2019 